\title{
INTERVENÇÃO PARA PROMOVER A QUALIDADE DO VÍNCULO MÃE-BEBÊ EM SITUAÇÃO DE NASCIMENTO PRÉ-TERMO
}

\author{
INTERVENTION TO IMPROVE THE QUALITY OF THE MOTHER-INFANT \\ BOND IN SITUATION OF PRE-TERM BIRTH
}

Evanisa Helena Maio de Brum*

Lígia Schermann ${ }^{* *}$

\begin{abstract}
Brum EHMd, Schermann L. Intervenção para promover a qualidade do vínculo mãe-bebê em situação de nascimento pré-termo. Rev Bras Crescimento Desenvolv Hum 2007; 17(2):12-23.

Resumo: O objetivo deste estudo foi avaliar a efetividade de uma intervenção precoce para promover a qualidade do vínculo mãe-bebê em situação de nascimento pré-termo. A amostra constou de 28 pares mãe-criança. 14 mães participaram da intervenção para promoção da qualidade do vínculo mãe-bebê através de videofilme (grupo experimental) e 14 mães assistiram a um videofilme sobre cuidados de rotina do bebê (grupo controle). Os videofilmes foram assistidos pelas mães no $2^{\circ}$ ou $3^{\circ}$ dia após o nascimento do bebê e, um mês após a alta hospitalar do bebê, foi realizada observação da interação mãe-bebê e aplicação do Mother and Baby Scales (MABS). Os resultados não diferenciaram o grupo experimental do grupo controle na quase totalidade das avaliações realizadas. O que leva a crer que somente uma intervenção em forma de videofilme, realizada no hospital, não seja suficiente para alterar positivamente a qualidade do vínculo mãe-bebê na população de pré-termos estudada. Sugere-se que sejam necessárias maior freqüência de intervenções hospitalares e a inclusão de intervenções domiciliares, bem como follow-ups longitudinais de médio prazo com pares de mães e bebês nascidos pré-termo.
\end{abstract}

Palavras-chave: Saúde coletiva. Nascimento pré-termo. Intervenção. Vínculo mãe-bebê.

\section{INTRODUÇÃO}

Os rápidos avanços científicos e tecnológicos nos cuidados com os recém-nascidos internados em Unidade de Terapia Intensiva Neonatal (UTIN) permitiram que, atualmente, um grande número destes recém-nascidos sobreviva ${ }^{1}$. Nos últimos 23 anos (de 1980 a 2003), os óbitos neonatais, em Porto Alegre, RS, diminuíram de 20 para 7,45 óbitos a cada 1.000 nascidos vivos ${ }^{2}$. Este panorama configura-se como uma realidade mundial ${ }^{3,4}$. Além da diminuição da mortalidade neonatal, autores apontam para o aumento da incidência da prematuridade e do baixo peso ao nas- cer $^{1,5,6}$, sendo que, entre outros fatores, o desenvolvimento da tecnologia da reprodutividade assistida contribui para a epidemia internacional de nascimentos múltiplos, prematuridade e baixo peso $^{6}$. Em Porto Alegre, RS, a incidência de prematuridade passou de 8,2 dos nascidos vivos, em 1999, para 10,8\% dos nascidos vivos em $2003^{7}$.

De forma geral, o aumento da incidência e a queda da mortalidade em pré-termos conduz, por conseguinte, a um aumento da morbidade, $\mathrm{o}$ que caracteriza uma transição epidemiológica. Portanto, os esforços, antes investidos na busca da sobrevivência, passam a focar a qualidade desta.

\footnotetext{
* Mestre em Saúde Mental Coletiva, Universidade Luterana do Brasil - ULBRA. Artigo baseado na dissertação de mestrado da primeira autora (bolsista CAPES) sob orientação da segunda. Rua João Abott 451 sala 204 - Petrópolis Porto Alegre - Rio Grande do Sul - Brasil CEP: 90460-150 Telefone: 0XX51.3061.78.20 ou 0XX51.9128.08.86 email: evanisa.brum@gmail.com

** Pós-Doutora em Psicologia, Uppsala Universitet, Suécia. Professora adjunta do Programa de Pós-Graduação em Saúde Coletiva e do Curso de Psicologia, Universidade Luterana do Brasil - ULBRA.
} 
Neste sentido a qualidade da relação mãebebê tem sido considerada fundamental para que ocorra um adequado desenvolvimento infantil ${ }^{8,9}$, onde os primeiros meses de vida são primordiais para o desenvolvimento da conduta de apego entre o bebê e sua mãe ${ }^{10,11}$. E, quando há o desenvolvimento de apego seguro, como postulam Ainsworthet et $\mathrm{al}^{12}$, tem-se a idéia de um importante fator no bom prognóstico do desenvolvimento afetivo, social e cognitivo de crianças.

Outro fator considerado positivo para a recuperação do bebê internado em UTIN, e que está diretamente relacionado com a formação de vínculos afetivos, é a presença e o envolvimento dos pais nos cuidados dispensados ao $\mathrm{RN}^{13-16}$. Se um bebê pré-termo é tocado durante sua permanência na UTIN (por 10 minutos, 3 vezes ao dia) este poderá apresentar níveis mais baixos de sono ativo, de atividade motora e de comportamento de estresse ${ }^{17}$, se o tocarmos e/ou conversarmos com ele, poderá apresentar menos falhas na respiração, ganho de peso, alteração da motilidade intestinal e do choro e um progresso mais rápido em algumas áreas de funcionamento cerebral ${ }^{11,18}$.

Portanto, se os pais estiverem presentes, envolvidos nos cuidados de seus filhos, e se estabelecerem uma relação de apego seguro, estes bebês terão uma maior e mais rápida probabilidade de recuperação. Neste ponto deparamo-nos com uma questão complexa, pois nesta situação de nascimento pré-termo tanto os bebês quanto os pais podem apresentar maior dificuldade em estabelecer e manter vínculos afetivos. Os bebês pré-termo são considerados de risco para o desenvolvimento em geral ${ }^{19,20}$, podem apresentar, entre outros fatores, maior predisposição a ter comportamento social menos competente devido à sua vulnerabilidade biológica $^{20,21}$. Os pais, por sua vez, frente ao nascimento de um bebê pré-termo, podem experienciar uma série de emoções negativas, sentem-se ansiosos, culpados, desesperançados, deprimidos e assustados ${ }^{1}$, sendo considerados prematuros para exercer suas funções parentais ${ }^{22}$.

Na literatura estudada a percepção da mãe, tanto em relação ao seu bebê quanto à sua capacidade de cuidados para com o bebê, influencia a qualidade da interação mãe-bebê. No caso das mães de crianças com risco médico neonatal, como as nascidas pré-termo, estas percepções podem ser negativamente atingidas. Neste sentido, promover um senso de competência nos cuidados maternos, bem como avaliá-los revelam-se importantes questões $^{23,24}$. Rabouam e Morales-Huet ${ }^{25}$ consideram a percepção materna sobre o bebê mais importante que qualquer outra característica do bebê, a qual influencia o sistema de cuidados da mãe, gerando a partir daí uma interação bidirecional, com influência tanto dos comportamentos do bebê quanto dos comportamentos maternos.

Portanto, neste contexto de nascimento prétermo, onde encontramos tanto uma maior incidência da prematuridade quanto um aumento da sobrevivência destes bebês, inúmeros estudos sobre intervenções precoces com bebês de risco têm sido realizados ${ }^{16}$, os quais buscam melhorar os efeitos negativos do nascimento pré-termo na parentalidade, na interação mãe-criança e, portanto, no desenvolvimento infantil ${ }^{1,26-30}$. Destaca-se que, a partir dessa concepção, os períodos neonatal e o pós-natal (do nascimento até 1 ano), têm sido considerados sensíveis para o desenvolvimento infantil e para a realização de intervenções ${ }^{31,32,33}$, as quais produzem ganhos que se mantêm a curto, médio e longo prazo ${ }^{34,35}$.

Patteson e Barnard ${ }^{27}$, em seu artigo de revisão sobre intervenção com pais de prematuros, citam dezenove estudos de intervenção que buscavam influenciar os resultados no comportamento e no desenvolvimento infantil através de apoio e informações aos pais. Dentre tais estudos, dezesseis apresentaram resultados positivos. Os autores referem que, através da análise destes estudos, encontraram evidências para afirmar que prover suporte e informação aos pais de prematuros pode alterar positivamente o curso do desenvolvimento infantil. Estudos mais recentes com pais de prematuros também têm pontuado intervenções de informação ${ }^{29,1,14}$ como fundamentais, mostrando ser uma ação efetiva não apenas na melhora do desenvolvimento infantil, mas também nas habilidades dos pais para exercer a paternalidade.

O objetivo deste estudo foi avaliar a efetividade de uma intervenção precoce para mães de bebês pré-termos internados em UTIN para promover a qualidade do vínculo mãe-bebê. Esta 
intervenção, realizada em vídeo-filme, consta de três momentos: familiarização com a UTIN, capacidades sociais do recém-nascido e depoimentos de profissionais de saúde sobre a importância do vínculo e da presença dos pais para a recuperação do bebê pré-termo. As intervenções, nestes períodos, constam como prioridades nas recomendações políticas ${ }^{36,37}$ e mostram-se, no campo da saúde coletiva, mais custo-efetivas ${ }^{38}$. Dentro desta concepção, é necessário identificar os fatores de risco para resultados adversos, como o nascimento pré-termo, e desenvolver intervenções para impedir e/ou minimizar tais efeitos $19,39,32,33,36,37$.

\section{MÉTODO}

A amostra do presente estudo constou de 28 bebês nascidos pré-termo (idade gestacional de 32 a 36 semanas) com necessidade de Tratamento Intensivo Neonatal (TIN) e suas mães. 14 mães participaram da intervenção (grupo experimental) e 14 mães compuseram o grupo controle. Os critérios de exclusão da amostra foram mães não residentes na Grande Porto Alegre, mães com histórico de internação psiquiátrica e idade inferior a 18 anos e crianças com malformação congênita, critérios mantidos no mínimo possível para a obtenção de uma amostra representativa.

As intervenções foram realizadas em dois Hospitais de Porto Alegre, RS, Brasil, no período de janeiro a novembro de 2004. Todas as mães e seus bebês que preenchiam os critérios de inclusão foram contatadas para participar do estudo, com exceção de 2 mães, com as quais não foi possível o contato por curta permanência hospitalar.

$\mathrm{O}$ projeto de pesquisa foi aprovado pelo Comitê de Ética da ULBRA e dos locais decoleta de dados (Parecer n ${ }^{\circ}$ 2003-118H e Parecer nº 042/04).

\section{Procedimentos}

A coleta de dados constou de 3 momentos. Primeiro houve a confecção dos videofilmes (controle e experimental), os quais foram elaborados especialmente para o estudo, tendo uma duração aproximada de 25 minutos cada um.
O filme experimental contém 3 partes: 1 . Familiarização: apresentação da UTIN com explicações sobre equipamentos e procedimentos, através do relato de profissionais da equipe multidisciplinar; 2. Capacidades sociais do bebê RN: relato de psicólogos enfocando e demonstrando as capacidades dos recém-nascidos - a termo e pré-termo - para formar vínculos. A seqüência do vídeo foi baseada na Escala de Avaliação do Comportamento Neonatal de Brazelton ${ }^{40}$ e no estudo de Wendland-Carro et al. ${ }^{41}$. Os itens focaram 4 áreas: (1) Capacidades perceptivas do RN, como orientação visual e auditiva. (2) Interação social: respostas do RN frente à fala e ao toque materno e respostas da mãe frente ao choro, vocalizações e expressões faciais da criança, (sensitividade e responsividade materna). (3) Organização do estado: se alerta, tranquiilo ou irritado. (4) Regulação do estado: reações da criança aos estímulos ou eventos agressivos do meio ambiente (consola-se, tranqüiliza-se, coloca a mão na boca, chora); 3. Depoimentos: depoimento de profissionais de saúde sobre bebês pré-termo e a importância da presença da mãe e do vínculo para a recuperação do bebê e depoimentos de mães com seus bebês de 9 meses que nasceram pré-termo, as quais mostraram fotos do período em que o bebê estava internado na UTIN.

O videofilme controle foi montado como um controle para o videofilme experimental, o qual também contém 3 partes: 1 . Familiarização: apresentação da UTIN com explicações sobre equipamentos e procedimentos, através do relato de profissionais da equipe multidisciplinar; 2. Cuidados durante as rotinas dos bebês; relato de profissionais da saúde sobre os cuidados de rotina com os bebês. A sequiência do vídeo focou 4 áreas: (1) amamentação, (2) banho, (3) cuidados com o coto umbilical, (4) aconselhamentos sobre a imunização da criança durante o período neonatal; 3 . Depoimentos: depoimento de profissionais de saúde sobre situações de cuidados de rotina de bebês pré-termo. As imagens da situação de banho e amamentação utilizadas para o videofilme controle foram retiradas do filme "Cuidados com o bebê: orientação e carinho em todas as fases", produzido pela Universidade Federal de São Paulo ${ }^{42}$.

Num segundo momento, ocorreram as in- 
tervenções experimental e controle em dia e hora agendadas com as mães. As mães foram encaminhadas, primeiro, para o Grupo Controle (GC) e, quando este foi preenchido, deu-se início ao Grupo Experimental (GE). Antes do início do trabalho de intervenção, as mães assinaram o termo de consentimento livre e esclarecido e responderam às questões da ficha de identificação. As intervenções experimental e controle ocorreram entre o $2^{\circ}$ ou $3^{\circ}$ dia após o nascimento, dentro das UTINs, em salas devidamente equipadas com televisão, videocassete e cadeiras confortáveis. A pesquisadora $(\mathrm{EB})$ permanecia junto às mães durante todo o período de intervenção, reservando 5 minutos entre cada uma das três partes dos videofilmes para comentários. Após a intervenção, as mães dos dois grupos receberam por escrito os itens apresentados nos respectivos vídeos para poderem observá-los em seus bebês.

Num terceiro momento, foi realizada a filmagem da interação mãe-bebê durante o banho do bebê e a situação de face a face em visita domiciliar, 30 dias após a alta hospitalar do bebê, bem como o preenchimento do Mother and Baby Scales (MABS).

Previamente ao início da coleta de dados, realizou-se um estudo piloto (Brum et al. ${ }^{43}, 2004$ ) para adequação dos procedimentos e instrumentos, bem como para o treinamento da equipe auxiliar da pesquisa, composta de seis alunos do curso de graduação de Psicologia da Universidade Luterana do Brasil - ULBRA/Canoas.

\section{Instrumentos de pesquisa}

A interação mãe-bebê foi avaliada com o protocolo de observação da interação mãebebê, elaborado por Schermann et al. ${ }^{44} \mathrm{e}$ traduzido por Schermann $^{45}$. O protocolo foi adaptado para o presente estudo e contém itens referentes ao comportamento da mãe, da criança e da díade (sintonia da interação). Os itens são pontuados em uma escala Likert de cinco pontos, em que escores mais altos equivalem à maior freqüência ou a um desempenho mais satisfatório. O tempo estabelecido para observação dos comportamentos foi de 5 minutos para a situação de facea-face e até 15 minutos para a situação de banho, seguido do registro da pontuação.
A interação mãe-bebê foi igualmente avaliada a partir de um segundo protocolo de frequiência de comportamentos interativos mãebebê. O protocolo contém medidas de tempo para o registro dos comportamentos da mãe, da criança e da díade em forma de check list ${ }^{46}$. O tempo estabelecido para observação dos comportamentos foi de 20 segundos, seguido, de pausa do filme por 30 segundos para o registro dos comportamentos observados durante 15 minutos do banho do bebê e 5 minutos da situação de face a face.

A avaliação com os dois protocolos foi realizada para cada uma das duas situações observadas (banho e face a face). A fidedignidade das pontuações foi obtida através do cálculo do percentual de acordo entre dois observadores independentes, o qual variou de $80 \%$ a $100 \%$, atingindo a média de $88 \%$ para o primeiro protocolo $^{47}$ e $85 \%$ (variação de $80 \%$ a $90 \%$ ) para o segundo protocolo, o que denota um acordo satisfatório.

O Mother and Baby Scales (MABS) ${ }^{48}$ foi utilizado para avaliar a percepção da mãe sobre o seu bebê e sobre sua capacidade de cuidados para com o bebê.

\section{Análise dos dados}

O programa SPSS 10.0 for Windows ${ }^{49}$ foi utilizado para realizar a análise descritiva (frequiência/percentual) da amostra, bem como para a comparação dos grupos estudados. Os testes estatísticos utilizados foram o teste do $\mathrm{X}^{2}$ para as variáveis nominais, o teste $t$ de Student para as variáveis intervalares, o Mann-Whitney para as variáveis ordinais e o teste de correlação de Spearman. O nível de significância estabelecido foi de $5 \%$. Os protocolos utilizados para as duas situações observadas (banho e face a face) foram agrupados para fins de análise.

\section{RESULTADOS}

Ao todo foram realizadas 41 intervenções em forma de videofilme, sendo 21 no Hospital 1 e 20 no Hospital 2. Do total de intervenções realizadas, 24 pertencem ao GC e 17 ao GE. 
Das 41 mães que participaram da intervenção, 28 concluíram a avaliação realizada com a visita domiciliar um mês após a alta, representando 68,3\% das mães contatadas. Das 13 mães que não concluíram a avaliação, 10 pertenciam ao GC (motivos relatados pelas mães foram: 2 bebês doentes; 2 mães não estavam pegando seus bebês no colo; 2 mães tomaram a decisão pessoal de não seguir participando do estudo; 1 mãe não foi localizada; 3 casos nos quais a filmagem realizada pela equipe apresentou falta de nitidez) e 3 destas mães pertenciam ao GE (motivos das perdas: 1 bebê doente, 1 mãe não localizada; 1 mãe tomou a decisão pessoal de não seguir participando do estudo). Não houve diferenças significativas entre as variáveis sociodemográficas e perinatais das díades que completaram o estudo e das que não completaram (idade, anos de estudo, número de filhos, tipo de parto, situação conjugal, ocupação, paridade, sexo do bebê, idade gestacional, peso ao nascer, apgar).

As tabelas 1 e 2 mostram as características da amostra estudada. Em relação às variáveis pe- rinatais, observa-se, na tabela 1 , que os bebês do GE apresentaram Apgar no $1^{\circ}$ e $5^{\circ}$ minuto significativamente menores do que os do GC, o que se reflete numa tendência a apresentarem maior Índice de Risco Médico Neonatal (IRMN). O teste de correlação de Spearman revelou que estas variáveis não interferiram nas medições do resultado da intervenção. As demais variáveis perinatais, bem como a idade da mãe e renda familiar e a escolaridade materna e paterna não diferiram significativamente entre os grupos. Cabe salientar que a média de anos de estudo materna e paterna, nos dois grupos, corresponde a pelo menos segundo grau completo.

A tabela 2 mostra que houve predominância de mães primíparas, casadas ou com união marital estável. A ocupação da mãe e do pai obteve maior incidência no nível II (trabalho técnico) no GC, enquanto que no GE esteve mais concentrada no nível I (trabalho braçal) para os pais ( $\mathrm{p}=0,072$ ) e no nível II para as mães (trabalho técnico). A maioria dos partos foi cesariana e o sexo dos bebês foi igualmente distribuído em ambos os grupos.

Tabela 1 - Características perinatais e sociodemográficas da amostra

\begin{tabular}{|c|c|c|c|c|c|c|}
\hline & \multicolumn{2}{|c|}{$\begin{array}{c}\text { GE } \\
(\mathrm{n}=14)\end{array}$} & \multicolumn{2}{|c|}{$\begin{array}{c}\mathrm{GC} \\
(\mathrm{n}=14)\end{array}$} & \multirow[b]{2}{*}{$\mathrm{t}$} & \multirow[b]{2}{*}{$\mathrm{p}^{\mathrm{a}}$} \\
\hline & Média & DP & Média & DP & & \\
\hline Idade gestacional & 33,79 & 1,37 & 34,57 & 1,09 & 1,680 & 0,105 \\
\hline Idade corrigida & 30,86 & 10,23 & 29,36 & 9,41 & $-0,404$ & 0,690 \\
\hline Idade cronológica & 49,29 & 13,80 & 45,07 & 6,60 & $-1,031$ & 0,312 \\
\hline Peso ao nascer & 217,71 & 587,66 & 214,29 & 514,28 & $-0,151$ & 0,881 \\
\hline Dias de internação & 18,93 & 13,74 & 14,00 & 7,47 & $-1,179$ & 0,249 \\
\hline Dias de oxigênio & 2,86 & 3,35 & 1,14 & 1,41 & $-1,766$ & 0,089 \\
\hline Dias de ventilação mecânica & 0,43 & 0,94 & 1,14 & 1,41 & 0 & 1,000 \\
\hline Apgar $1^{\circ}$ minuto & 6,29 & 2,09 & 7,71 & 1,49 & 2,082 & 0,047 \\
\hline Apgar $5^{\circ}$ minuto & 8,29 & 0,91 & 8,93 & 0,47 & 2,336 & 0,030 \\
\hline IRMN & 2,00 & 0,55 & 1,57 & 0,65 & $-1,883$ & 0,071 \\
\hline Consultas pré-natal & 7,43 & 2,53 & 8,36 & 3,50 & 0,804 & 0,429 \\
\hline Idade da mãe & 27,57 & 7,27 & 28,00 & 5,56 & 0,175 & 0,862 \\
\hline Anosde estudoda mãe & 11,72 & 2,48 & 11,36 & 3,39 & $-0,314$ & 0,756 \\
\hline Número de filhos & 1,43 & 0,65 & 1,36 & 0,50 & $-0,328$ & 0,746 \\
\hline Anos de estudo do pai & 11,93 & 2,46 & 10,79 & 3,12 & $-1,076$ & 0,292 \\
\hline Renda familiar total (SMN) & 6,91 & 3,01 & 11,17 & 13,53 & 1,150 & 0,260 \\
\hline
\end{tabular}

a: Teste t-student ( $\mathrm{gl}=26)$; GC: Grupo Controle; GE: Grupo Experimental; SMN: Salário Mínimo Nacional; IRMN: Índice de Risco Médio Neonatal 
Tabela 2 - Características sóciodemográficas da amostra

\begin{tabular}{|c|c|c|c|c|c|c|}
\hline & \multicolumn{2}{|c|}{$\begin{array}{c}\mathrm{GE} \\
(\mathrm{n}=14)\end{array}$} & \multicolumn{2}{|c|}{$\begin{array}{c}\mathrm{GC} \\
(\mathrm{n}=14)\end{array}$} & \multirow[b]{2}{*}{$x^{2}$} & \multirow[b]{2}{*}{$\mathrm{p}^{\mathrm{a}}$} \\
\hline & $\mathrm{n}$ & $\%$ & $\mathrm{n}$ & $\%$ & & \\
\hline Sexo masculino do RN & 8 & 57,1 & 7 & 50 & 0,144 & 0,705 \\
\hline \multicolumn{7}{|l|}{ Itens da mãe } \\
\hline Primipara & 9 & 64,3 & 9 & 64,3 & 0 & 1 \\
\hline Parto Cesária & 11 & 78,6 & 13 & 92,9 & 1,167 & 0,280 \\
\hline Casada ou união estável & 13 & 92,9 & 13 & 92,9 & 0 & 1 \\
\hline Ocupação materna ${ }^{\mathrm{b}}$ & & & & & 2,803 & 0,423 \\
\hline II & 5 & 35,7 & 8 & 57,1 & & \\
\hline III & 2 & 14,3 & 2 & 14,3 & & \\
\hline IV & 2 & 14,3 & 0 & 0 & & \\
\hline \multicolumn{7}{|l|}{ Itens do pai } \\
\hline \multicolumn{7}{|l|}{ Ocupação paterna $^{\mathrm{b}}$} \\
\hline I & 9 & 46,4 & 4 & 28,6 & 5,256 & 0,072 \\
\hline II & 3 & 21,4 & 9 & 64,3 & & \\
\hline III & 2 & 14,3 & 1 & 7,1 & & \\
\hline
\end{tabular}

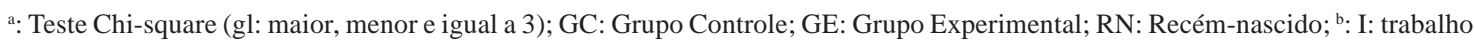
braçal; II: trabalho técnico; III: trabalho de nível superior; IV: desempregado.

Tabela 3 - Distribuição dos itens avaliados no protocolo de observação da interação mãe-bebê- 0-6 meses para os grupos controle e experimental

\begin{tabular}{|c|c|c|c|c|c|c|c|c|}
\hline & \multicolumn{3}{|c|}{$\begin{array}{c}\text { GE } \\
(n=14)\end{array}$} & \multicolumn{3}{|c|}{$\begin{array}{c}\mathrm{GC} \\
(\mathrm{n}=14)\end{array}$} & \multirow[b]{2}{*}{$\mathrm{U}$} & \multirow[b]{2}{*}{$\mathrm{p}^{\mathrm{a}}$} \\
\hline & Mediana & Minímo & Máximo & Mediana & Minímo & Máximo & & \\
\hline \multicolumn{9}{|l|}{ Itens da mãe } \\
\hline Verbalização da mãe & 3,50 & 2,50 & 4,00 & 4,00 & 2,50 & 4,50 & 52,00 & 0,024 \\
\hline Contato visual & 3,50 & 3,00 & 4,00 & 3,50 & 3,00 & 4,00 & 95,50 & 0,900 \\
\hline Contato corporal & 4,00 & 2,50 & 4,50 & 3,50 & 2,50 & 4,50 & 84,00 & 0,507 \\
\hline Expressão de afeto positivo & 3,50 & 3,00 & 4,50 & 4,00 & 2,50 & 5,00 & 58,50 & 0,061 \\
\hline Eficiência para consolar & 3,50 & 2,00 & 4,00 & 3,50 & 2,50 & 4,50 & 25,00 & 0,809 \\
\hline Reação ao choro & 4,00 & 3,50 & 4,50 & 3,50 & 2,50 & 4,50 & 12,00 & 0,066 \\
\hline Resposta ao comport. social do bebê & 4,00 & 3,00 & 4,50 & 4,00 & 3,00 & 4,50 & 94,00 & 0,840 \\
\hline Intensidade da resposta & 3,50 & 2,50 & 4,50 & 4,00 & 2,50 & 5,00 & 77,00 & 0,324 \\
\hline Expressão de afeto positivo & 4,50 & 2,50 & 5,00 & 4,50 & 3,50 & 5,00 & 94,50 & 0,864 \\
\hline Sensitividade & 3,50 & 2,50 & 4,50 & 3,75 & 2,50 & 4,00 & 95,50 & 0,904 \\
\hline Atenção geral & 5,00 & 4,50 & 5,00 & 4,75 & 3,50 & 5,00 & 60,00 & 0,034 \\
\hline Menos comport. Exagerado & 4,50 & 3,50 & 5,00 & 4,00 & 3,00 & 5,00 & 65,00 & 0,114 \\
\hline \multicolumn{9}{|l|}{ Itens do bebê } \\
\hline Olhares para a mãe & 2,50 & 1,00 & 3,00 & 2,50 & 1,50 & 3,00 & 94,50 & 0,867 \\
\hline Respostas às comunicações & 3,00 & 1,00 & 4,00 & 3,00 & 2,00 & 4,00 & 81,00 & 0,414 \\
\hline Respostas positivas & 3,00 & 1,00 & 3,50 & 3,00 & 1,50 & 3,50 & 94,50 & 0,866 \\
\hline Tentativa de contato & 2,50 & 1,50 & 3,50 & 2,50 & 2,00 & 3,00 & 90,00 & 0,700 \\
\hline Quantidade de sorrisos & 1,00 & 1,00 & 2,00 & 1,00 & 1,00 & 1,00 & 84,00 & 0,150 \\
\hline Bom humor & 3,50 & 2,00 & 5,50 & 4,00 & 2,50 & 5,00 & 81,50 & 0,437 \\
\hline Quantidade de vocalizações & 2,50 & 1,50 & 3,00 & 2,50 & 2,00 & 3,00 & 91,50 & 0,751 \\
\hline Quantidade de choro & 4,00 & 2,00 & 5,00 & 4,00 & 2,00 & 5,00 & 87,00 & 0,607 \\
\hline Estado predominante & 4,50 & 3,50 & 5,00 & 5,00 & 4,00 & 5,00 & 58,00 & 0,050 \\
\hline \multicolumn{9}{|l|}{ Itens da diade } \\
\hline Sintonia da interação & 3,00 & 2,00 & 4,50 & 2,50 & 1,00 & 3,50 & 73,00 & 0,237 \\
\hline
\end{tabular}

a: Teste de Mann-Whitneyt; GC: Grupo Controle; GE: Grupo Experimental. 
Frente ao convite para participarem do estudo, 5 mães recusaram-no devido ao alto nível de estresse ou exaustão. Estas mães representam $12,1 \%$ da amostra total. De acordo com Melnyk et al., (2001), esta razão de recusa está abaixo da encontrada em estudos com mães de pré-termos, nos quais, a razão variou de $18 \%$ a $40 \%$. As variáveis sociodemográficas e perinatais não diferiram significativamente entre as díades que participaram do estudo e as que se recusaram a participar.

A Tabela 3 mostra os resultados obtidos na comparação entre o GE e o GC para as variáveis referentes à interação mãe-bebê através do teste de Mann-Whitney. Somente três itens revelaramse significativos estatisticamente, sendo que apenas um com diferença favorável para o GE: as mães do GE mostraram-se mais atentas aos seus bebês quando comparadas as do GC, as mães do GC verbalizaram mais para seus bebês quando comparadas as mães do GE e os bebês do GC mostraram-se mais alertas que os bebês do GE.
Como pode ser observado na Tabela 4, o levantamento estatístico do protocolo de freqüência de comportamentos interativos mãe-bebê não revelou diferenças significativas entre os dois grupos.

Igualmente, o levantamento estatístico do MABS não revelou diferenças significativas entre os dois grupos, tanto no que se refere aos sentimentos maternos em relação a si e ao bebê quanto à alimentação do bebê (Tabela 5).

Ao cruzar as variáveis sociodemográficas com as variáveis da interação mãe-bebê detectouse que as mães mais jovens comparativamente com mães mais velhas apresentaram maior sintonia na interação com seus filhos $(\mathrm{t}=2,157$; $\mathrm{p}=0,040$ ). Igualmente, observou-se uma tendência dos bebês com índice de Apgar maior no $5^{\circ}$ minuto $(\mathrm{M}=8,74 ; \mathrm{DP}=0,69)$ apresentarem mais respostas positivas durante a interação com suas mães ( $\mathrm{t}=$ $2,01 ; \mathrm{p}=0,055)$ do que aqueles que obtiveram Apgar menor.

Tabela 4 - Distribuição nos itens pontuados no protocolo de frequência de comportamentos interativos mãe-bebê para os grupos controle e experimental

\begin{tabular}{|c|c|c|c|c|c|c|}
\hline & \multicolumn{2}{|c|}{$\mathrm{GE}(\mathrm{n}=14)$} & \multicolumn{2}{|c|}{$\mathrm{GC}(\mathrm{n}=14)$} & \multirow[b]{2}{*}{$\mathrm{t}$} & \multirow[b]{2}{*}{$\mathrm{p}^{\mathrm{a}}$} \\
\hline & Média & $\mathrm{DP}$ & Média & DP & & \\
\hline Mãe olha para a criança & 31 & 5,71 & 33,29 & 9,02 & 0,801 & 0,431 \\
\hline Criança olha para a mãe & 12,21 & 7,52 & 10,14 & 6,2 & $-0,795$ & 0,434 \\
\hline Olhar mútuo & 7,57 & 5,23 & 6,79 & 5,78 & $-0,377$ & 0,709 \\
\hline Mãe verbaliza para a criança & 31,93 & 6,4 & 36,57 & 7,33 & 1,784 & 0,086 \\
\hline Criança vocaliza para a mãe & 19,43 & 6,07 & 22,21 & 7,53 & 1,078 & 0,291 \\
\hline Verbalização mútua & 4,29 & 4,07 & 6,71 & 5,21 & 1,375 & 0,181 \\
\hline Mãe sorrí para a criança & 4,21 & 4,49 & 4,57 & 4,16 & 0,218 & 8,829 \\
\hline Criança chora & 17,86 & 9,68 & 20,36 & 9,9 & 0,675 & 0,505 \\
\hline
\end{tabular}

a: Teste t-Student ( $\mathrm{gl}=26)$; GC: Grupo Controle; GE: Grupo Experimental.

Tabela 5 - Distribuição nos itens avaliados no MABS para o grupo experimental e controle

\begin{tabular}{|c|c|c|c|c|c|c|}
\hline & \multicolumn{2}{|c|}{$\mathrm{GE}(\mathrm{n}=14)$} & \multicolumn{2}{|c|}{$\mathrm{GC}(\mathrm{n}=14)$} & \multirow[b]{2}{*}{$\mathrm{t}$} & \multirow[b]{2}{*}{$\mathrm{p}^{\mathrm{a}}$} \\
\hline & Média & DP & Média & DP & & \\
\hline \multicolumn{7}{|c|}{ Sentimentos maternos em relação a sí e ao bebê } \\
\hline Bebê alerta e responsivo & 33,64 & 5,06 & 31,29 & 5,68 & $-1,16$ & 0,257 \\
\hline Bebê agitado / humor irregular & 27,57 & 9,8 & 26,86 & 12,57 & $-0,168$ & 0,868 \\
\hline Falta confiança mãe cuidado & 12,57 & 7,85 & 14,43 & 10,48 & 0,531 & 0,600 \\
\hline Bebê fácil & 6,57 & 2,9 & 7,71 & 3,69 & 0,911 & 0,371 \\
\hline Confiança global da mãe & 4,43 & 2,98 & 4,43 & 3,78 & 0 & 1 \\
\hline \multicolumn{7}{|l|}{ Alimentação do bebê } \\
\hline Bebê alerta durante alimentação & 15,71 & 4,43 & 13,5 & 4,45 & $-1,32$ & 0,198 \\
\hline Bebê irritadodurante alimentação & 4,86 & 4,19 & 8 & 6,4 & 1,538 & 0,136 \\
\hline Falta confiança mãe alimentação & 5,82 & 3,22 & 7,8 & 4,87 & 1,11 & 0,281 \\
\hline
\end{tabular}

a: Teste t-Student ( $\mathrm{gl}=26)$; GC: Grupo Controle; GE: Grupo Experimental. 


\section{DISCUSSÃO}

O presente estudo propôs-se a contribuir com uma metodologia de intervenção preventiva precoce em saúde coletiva para as mães de bebês pré-termo internados em UTIN. Nossa hipótese inicial de que a intervenção em forma de videofilme para mães de bebês pré110 termos internados em UTIN poderia melhorar a qualidade do vínculo da mãe com seu bebê não foi encontrada.

Apesar do dado inesperado dos bebês do GE apresentarem-se mais comprometidos do ponto de vista médico que os do GC (Apgar mais baixo no $1^{\circ}$ e $5^{\circ}$ minuto de vida), o teste de correlação de Spearman revelou que estas variáveis não interferiram nas medições do resultado da intervenção.

Frente a estes dados, nossa primeira e mais óbvia hipótese para a não efetividade da intervenção é que o modelo de intervenção adotado e baseado no estudo de Wendland-Carro et. al. ${ }^{41}$, delineado para melhorar a relação mãe-bebê de crianças a termo e sadias, precisa ser mais bem pensado para população de pré-termos, portanto, esta população requer outro tipo de intervenção.

A segunda hipótese para a não efetividade da intervenção seria com relação ao conteúdo do vídeo apresentado para o grupo controle, o banho e a amamentação são situações de rotina que estimulam o toque no bebê e a proximidade da díade, podendo ter produzido um efeito benéfico na interação. Para testar esta suposição seria necessária a inclusão de um terceiro grupo, o qual não receberia nenhum tipo de intervenção.

A terceira hipótese tem relação com a freqüência e o local da intervenção. Nossa intervenção foi realizada em uma única vez no hospital. Entre os estudos delineados para melhorar o desenvolvimento infantil através da influência do comportamento dos pais, encontramos resultados positivos com apenas uma intervenção realizada no hospital, como a pesquisa realizada por Wendland-Carro et al. ${ }^{41}$. Em revisão de 19 estudos sobre intervenção com prematuros, Patteson e Barnard $^{27}$ salientam que os únicos três estudos em que os resultados não foram positivos apresentavam duas características comuns: intervenções curtas e realizadas somente no hospital.
Nos demais estudos com resultados positivos, as intervenções foram domiciliares ou domiciliares e hospitalares, e apresentavam também maior frequiência de contatos com os pais, com exceção de dois estudos com intervenções curtas. Em outros estudos de intervenção que enfocaram a sensitividade e a responsividade dos pais de prétermos e que obtiveram resultados positivos, podemos identificar uma maior freqüência das intervenções e, quanto ao local da realização da intervenção, constatamos o que Patteson e Barnard ${ }^{27}$ salientaram: 3 intervenções no hospital e 1 em casa ${ }^{1}, 8$ sessões de intervenção em casa ${ }^{29}$, 20 sessões de intervenção em casa ${ }^{30}$, e 7 sessões de intervenção no hospital e 4 em casa ${ }^{50}$. Brown et al. ${ }^{29}$, em sua pesquisa, encontraram forte relação entre o tempo que as mães assistiram à intervenção em forma de videofilme e o resultado positivo da intervenção.

A quarta hipótese para o fracasso da intervenção refere-se ao período da avaliação. Pensamos que 30 dias depois da alta possa ter sido um tempo curto para avaliar o efeito da intervenção, portanto, seria necessária uma nova avaliação a longo prazo. Alguns estudos de intervenção com prematuros, como o "Vermont Intervention Program for Low-birthweight Infants", realizado por Achenbach et al. ${ }^{5}$, e o estudo de Sajaniemi et al. ${ }^{30}$ mostraram resultados efetivos somente a longo prazo. Na pesquisa de Achenbach et al. ${ }^{50}$ (1990), foram realizadas intervenções com o objetivo de auxiliar os pais na transição do bebê do hospital para casa, a intervenção enfocou a sensitividade e a responsividade materna. As crianças do grupo experimental apresentaram escores mentais mais elevados que as do grupo controle aos 3,4 e 7 anos $(\mathrm{p}<0,05)$, o que nas avaliações anteriores (6, 12 e 24 meses) não havia acontecido. Sajaniemi et al. ${ }^{30}$ realizaram 20 intervenções de terapia ocupacional com 100 recém-nascidos prematuros de baixo peso, também com o objetivo de melhorar a sensitividade e a responsividade dos pais para com suas crianças. A avaliação aos 2 anos de idade não apresentou resultado positivo, os pesquisadores pensaram que a falta de resultado positivo não necessariamente significava que a intervenção não tivesse produzido efeito, 
desta forma, outra avaliação foi realizada aos 4 anos com a obtenção de resultado positivo para o grupo que recebeu a intervenção.

Os autores dos dois estudos citados anteriormente salientam a importância de realizar avaliações longitudinais com a mesma amostra para que os resultados de intervenções com crianças nascidas prematuras e com baixo peso sejam realmente avaliados de forma efetiva e que, em alguns casos, as intervenções precoces podem ter um efeito favorável a longo prazo e, até mesmo, apresentar efeito aumentado a longo prazo.

$\mathrm{O}$ estudo realizado por Brown et al. ${ }^{29}$ para mães de risco corrobora para a questão citada. Os pesquisadores identificaram a subamostra de crianças nascidas pré-termos e encontraram que as mães de pré-termos do grupo experimental foram as únicas que não apresentaram ganhos significativos com a intervenção na avaliação realizada aos 6 meses, mas obtiveram escores significativos $(\mathrm{p}<0,025)$ na avaliação realizada aos 12 meses. O estudo de intervenção para crianças nascidas prematuras e baixo peso, realizado por Melnyk et al. ${ }^{1}$ também encontrou aumento do efeito da intervenção a longo prazo. As avaliações realizadas aos 3 e 6 meses apresentaram resultados significativos, porém os pesquisadores constataram que a diferença foi ampliada positivamente na avaliação realizada aos 6 meses para as mães do grupo experimental.

Outro fato que justificaria uma nova avaliação é que, em 30 dias após a alta, as condições neonatais ainda se refletem de forma decisiva no estado do bebê, e que quanto mais grave o estado de saúde do bebê mais atingido este será pelo risco perinatal, como no caso dos bebês do GE de nossa pesquisa. Sajaniemi et al. ${ }^{30}$ salientam que tem sido repetidamente mostrado que fatores de risco perinatal têm um efeito diminuído ao longo do desenvolvimento, enquanto os efeitos do ambiente passam a ter preponderância. Desta forma, podemos supor que, com o tempo, os bebês do GE estariam sob uma influência menor do seu estado de nascimento para a realização de uma nova avaliação. Melnyk et al. ${ }^{1}$ também não encontraram diferenças na interação da díade entre o grupo que recebeu a intervenção e o grupo controle, referem que outros estudos de intervenção não têm encontrado efeitos positivos da intervenção com prematuros até 36 meses de idade.

Partindo para os resultados encontrados no levantamento estatístico, encontramos as mães do GE mais atentas $(\mathrm{p}=0,034)$ e tendendo a reagir mais ao choro de seus bebês $(\mathrm{p}=0,066)$, o que poderia ser um pequeno efeito da intervenção ou ter relação com o estado de saúde mais grave destes bebês quando comparados aos do GC. Os bebês do GC mostraram-se mais alertas $(\mathrm{p}=0,050)$ do que os do GE, o que também parece ter relação com o melhor estado de saúde destes bebês. Primeiro, porque a imaturidade do pré-termo faz com que este apresente maior dificuldade de organizar o seu estado, de ficar mais tempo alerta, resultando em menos tempo de interação com os pais e menos resposta nas interações quando comparado aos bebês nascidos a termo ${ }^{51,21}$, sendo que quanto mais grave o estado de saúde do prétermo mais ele é atingido por estas dificuldades. Segundo, porque a situação de prematuridade configura-se como um fator de risco para a interação, onde tanto a criança encontra-se vulnerável como a mãe, e quanto mais grave é o estado de saúde da criança mais o comportamento materno pode ser afetado negativamente ${ }^{27}$.

Uma outra hipótese para os bebês do GC mostrarem-se mais alertas pode ter relação com o fato destas mães também falarem mais com seus bebês ( $\mathrm{p}=0,024)$. Novamente, a melhor condição de saúde destes bebês provavelmente permite que possam estar mais alertas e conseqüentemente que possam responder mais durante a interação com suas mães, o que, por sua vez, pode desencadear uma melhor resposta materna.

O estudo apresentou limitações que fazem com que os resultados encontrados sejam uma possibilidade, porém, a maior limitação do estudo foi o pequeno tamanho da amostra, o que resultou em um limitado poder de detectar diferenças estatisticamente significativas entre os grupos (erro tipo II). O resultado do presente estudo revela a realidade da amostra destes hospitais neste período, portanto, não apresenta o poder de generalização.

Como contribuição deste trabalho, a intervenção experimental em forma de videofilme seguirá sendo realizada na UTIN de um dos 
hospitais em que a pesquisa foi realizada, incorporando-se à rotina da equipe, ou seja, todas as mães que tiverem seus filhos internados assistirão à intervenção.

\section{CONSIDERAÇÕES FINAIS}

Não há dúvidas que intervenções de prevenção precoce podem alterar positivamente a trajetória do desenvolvimento de crianças com risco biológico, como as nascidas pré-termo, e que intervenções em forma de videofilme apresentam um duplo benefício para a saúde coletiva: facilidade de aplicação e baixo custo. Porém, o resultado do presente estudo leva a crer que somente uma intervenção de prevenção em forma de videofilme, realizada no hospital, não seja suficiente para alterar positivamente a qualidade da interação mãe-bebê na população de pré-termos estudada. Pensamos que seja necessário maior freqüência das intervenções hospitalares e a inclusão de intervenções domiciliares, bem como o seguimento de avaliações longitudinais da amostra. Qual o número de intervenções e o tempo necessário para acompanharmos estas mães e obtermos resultados positivos são questões que seguem em aberto.

\begin{abstract}
The present study aimed to evaluate an early preventive intervention to improve the quality of the mother-infant bond in situation of preterm birth. The sample comprised 28 motherinfant pairs. Fourteen pairs participated in the intervention for promotion of quality in the mother-infant bond through a video film (experimental group), and fourteen pairs watched a video on routine care for infants (control group). The videos were watched by the mothers on the $2^{\text {nd }}$ or $3^{\text {rd }}$ day after the infant's birth. One month after the infant's discharge from hospital, an evaluation of the interventions was conducted by observing the mother-infant interaction and by applying the Mother and Baby Scales (MABS). Results did not differentiate the experimental group from the control group in nearly all the evaluations performed. These results suggest that only one intervention using a video film, carried out at the hospital, was not enough to favorably change the quality of the mother-infant bond in the studied population of preterm infants. It is suggested that, for early preventive interventions with preterm infants, a greater frequency of hospital interventions and also inclusion of home interventions are necessary, as well as middle-term cross-sectional follow-ups.
\end{abstract}

Keywords: Public health. Preterm birth. Intervention. Mother-infant bond.

\section{REFERÊNCIAS}

1. Melnyk B et al. Improving cognitive development of low birth weight premature infants with the COPE program: a pilot study of the benefit of early NICU intervention with mothers. Research in Nursing \& Health. 2001;24:373-89.

2. SIM Série histórica dos coeficientes de mortalidade neonatal, pós-neonatal e infantil, Rio Grande do Sul e Porto Alegre, 1980-2003. Porto Alegre: SIM/EI/CGVS/SMS/PMPA; 2004.

3. Kleinman J. The epidemiology of low birthweight. In: Friedman SL, Sigman MD, organizadores. The psychological development of low birthweight children. Norwood, NJ: Ablex Publishing Corporation; 1992. p. 25-35.
4. Mccormick MC. Advances in neonatal intensive care technology and their possible impact on the development of low-birthweight infants. In: Friedman SL, Sigman MD, organizadores. The psychological development of low birthweight children. Norwood, NJ: Ablex Publishing Corporation; 1992. p. 37-60.

5. Horta $\mathrm{B}$ et al. Baixo peso ao nascer em duas coortes de base populacional no Sul do Brasil. Cadernos de Saúde Pública. 1996;12(1):27-31.

6. Grimes DA, Schulz KF. Descriptive studies: what they can and cannot do. The Lancet. 2002; 359:145-9.

7. SINASC. Distribuição dos nascidos vivos residentes em Porto Alegre segundo variáveis relacionadas à gestação e parto e do recém nascido, Porto Alegre, RS, 1999-2003. Porto Alegre: SINASC/EI/CGVS/SMS/PMPA; 2004. 
8. Bowlby J. Uma base segura: Aplicações clínicas da teoria do apego. Porto Alegre: Artes Médicas; 1989.

9. Schaffer HR. Social development. Oxford: Blackwell Publishers; 1996.

10. Claussen AH, Crittenden PM. Maternal sensitivity. In: Claussen AH, Crittenden PM , organizadores. The organization of attachment relationships: maturation, culture and context. Nova York: Cambridge University Press; 2000. p.115-22.

11. Klaus MH, Kennell J. Vínculo: Construindo as bases para um apego seguro e para a independência. Porto Alegre: Artes Médicas; 2000.

12. Ainsworth MD, Blehar MC, Waters E, Wall S. Patterns of attachment: A psychological study of the strange situation. New Jersey: Lawrence Erlbaum Associates Publishers Hillsdale; 1978.

13. Rauh V, Brennan J. An interactionist perspective on interventions with lowbirthweight infants. In: Friedman SL, Sigman MD, organizadores. The psychological development of low birthweight children. Norwood, NJ: Ablex Publishing Corporation; 1992. p.435-70.

14. Auslander A, Netzer D, Arad I. Parental anxiety following discharge from hospital of their very low weight infants. Family Relations. 2003;52:12-21.

15. Brum EHM, Schermann L. Vínculos iniciais e desenvolvimento infantil: abordagem teórica em situação de nascimento de risco. Ciência \& Saúde Coletiva. 2004;9(2):43-55.

16. Brum EHM, Schermann L. Intervenções frente ao nascimento prematuro: uma revisão teórica. Scientia Medica. 2005; 15(1):60-67.

17. Harrison L, Williams A, Berbaum M, Stem J, Leeper J. Physiologic and behavioral effects of gentle human touch on preterm infants. Research in Nursing \& Health. 2000;23:435-46.

18. Brazelton TB. O desenvolvimento do apego: Uma família em formação. Porto Alegre: Artes Médicas; 1988.

19. Boshi R. La prévention des troubles psychiques chez l'enfant et l'adolescent. Quand faut-il intervenir? Paris: L'Harmattan; 2000.

20. Schermann L. Considerações sobre a interação mãe-criança e o nascimento pré-termo. Temas em Psicologia da SBP. 2001; 9(1):55-61.
21. Als H. Earliest intervention for preterm infants in the newborn intensive care unit. In: Guralnick M. The effectiveness of early intervention. Baltimore: Paul H. Brookes Pub; 1997. p. 47- 76.

22. Als H. Individualized, family-focused developmental care for very low-birthweight preterm infant in the NICU. In: Friedman SL, Sigman MD. The psychological development of low birthweight children. Norwood, NJ: Ablex Publishing Corporation; 1992. p.341-87.

23. Stern M, Karraker K, Sopko A, Norman S. The prematurity stereotype revisited: Impact on mothers' interactions with premature and full-term infants. Infant Mental Health Journal. 2000;21(6):495-509.

24. Pridham K, Lin C, Brown R. Mothers' evaluation of their care giving for premature and full-term infants through the first year: contributing factors. Research in Nursing \& Health. 2001;24:157-69.

25. Rabouam C, Morales-Huet M. Cuidados parentais e vinculação. In: Guedeney N, Guedeney A (orgs). Vinculação: conceitos e aplicações. Lisboa: CLIMEPSI; 2003. p.71-85.

26. Field TM, Widmayer SM, Stringer S, Ignatoff E. Teenage, lower class black mothers and their preterm infants: an intervention and developmental follow-up. Child Development. 1980;51:426-436.

27. Patteson DRN, Barnard KE. Parenting of low birth weight infants: a review of issues and interventions. Infant Mental Health Journal. 1990;11(1):37-56.

28. Beckwith L, Rodning C. Evaluating effects of intervention with parents of preterm infants. In: Friedman SL, Sigman MD ,organizadores. The psychological development of low birthweight children. Norwood, NJ: Ablex Publishing Corporation; 1992. p.389-409.

29. Brown M, Yando R, Rainforth M. Effects of an at-home video course on maternal learning, infant care and infant health. Early Child Development and Care. 2000; 160:47-65.

30. Sajaniemi $\mathrm{N}$ et al. Cognitive performance and attachment patterns at four birth weight infants after early intervention. European Child \& Adolescent Psychiatry. 2001; 10:1229.

31. Guralnick M. Second generation research in the field of early intervention. In: Guralnick M. The effectiveness of early intervention. Baltimore: Paul H. Brookes Pub; 1997. p.3-22. 
32. Cowen E, Durlak J. Social policy and prevention in mental health. Development and Psychopathology. 2000;12:815-34.

33. Dawson G, Ashman S, Carver L. The role of early experience in shaping behavioral and brain development and its implications for social policy. Development and Psychopathology. 2000;12:695-712.

34. Campbell F, Ramey C. Effects of early intervention on intellectual and academic achievement: a follow-up study of children from low-income families. Child Development, 1994; 65:684-98.

35. Blair C, Ramey C. Early intervention for lowbirth weight infants and the path to second generation research. In: Guralnick M. The effectiveness of early intervention. Baltimore: Paul H. Brookes Pub; 1997. p.77-97.

36. Who. Setting the WHO agenda for mental health. Bulletin of World Health Organization. 2000; 78(4):500-14.

37. Organização Mundial de Saúde. Saúde mental: novos conhecimentos, novas esperanças. Genebra: OMS; 2001.

38. Luthar S, Cicchett D. The construct of resilience: Implications for interventions and social policies. Development and Psychopathology. 2000;12:857-85.

39. Costelo E, Angold A. Developmental psychopathology and public health: Past, present and future. Development and Psychopathology. 2000;12:599-618.

40. Brazelton TB, Nugent JK. Neonatal behavioral assessment scale. London: Lavenham Press; 1995.

41. Wendland-Carro J, Piccinini CA, Millar WS. The role of an early intervention on enhancing the quality of mother-infant interaction. Child Development. 1999;70(3):713-21.

42. Universidade Federal de São Paulo. Cuidados com o bebê: orientação e carinho em todas as fases. São Paulo: UFSP; 1 fita de vídeo (75 mim), VHS; 1996.

43. Brum EHM et al. Intervenção com mães de bebês prematuros: estudo piloto [resumo]. In: Anais do V Fórum de Pesquisa Científica e Tecnológica e X Salão de Iniciação Científica; 2004; Canoas, Brasil. Canoas: ULBRA; 2004. p. 232.

44. Schermann L, Bohlin G, Hagekull B. Interaction between mother and pre-term infant at 34 weeks post-conceptional age. Early Development and Parenting. 1994;3(3):171-80.

45. Schermann L. Observação do comportamento social. Psico. 1999;30(2):95-102.

46. Bakeman R, Gotiman JM. An introduction to sequential analysis. Cambridge: Cambridge University Press; 1986.

47. Brum EHM, Schermann L, Costa M, Martins M. Intervenção precoce para mães de bebês nascidos pré-termo [resumo]. In: Anais do IV Fórum de Pesquisa Científica e Tecnológica IX e Salão de Iniciação Científica; 2003; Canoas, Brasil. Canoas: ULBRA; 2003. p. 232.

48. Wolke D, Robert J. Mother and Baby Scales (MABS). In: Brazelton T, Nugent J. Neonatal Behavioral Assessment Scale. London: Lavenham Press; 1987. p. 135-7.

49. SPSS. Statistical Package [computer program]. Version 9.0. Chicago: SPSS; 1998.

50. Achenbach T, Phares V, Howell C, Rauh V, Nurcombe B. Seven-Years outcome of the Vermont intervention program for low birthweight infants. Child Development. 1990;61:1672- 81.

51. Eckerman O, Oehler M. Very low birthweight newborns and parents as early social partners. In: Friedman SL, Sigman MD, organizadores. The psychological development of low birthweight children. Norwood, NJ: Ablex Publishing Corporation; 1992. p.91-124.

Recebido em 02/04/2007 Aprovado em 26/04/2007 